\title{
A Method of WSN and Sensor Cloud System to Monitor Cold Chain Logistics as Part of the IoT Technology
}

\author{
Abel Avitesh Chandra* and Seong Ro Lee \\ Department of Electronics Engineering, Mokpo National University, South Korea \\ abelavit@yahoo.com
}

\begin{abstract}
The Internet of Things (IoT) is a new evolution in technological advancement taking place in the world today. This paradigm allows physical world objects in our surroundings to be connected to the Internet. This idea comes to life by utittsing two architecture; the Sensing Entity in the environment that collects data and connects itself to the cloud and the Cloud Service that hosts the data from the environment. The combination of wireless sensor networks and cloud computing is becoming a popularstrategy for the IoT era. The cold chain requires controlled environment for sensitive products in order for them to be fit for use. The monitoring process is the only assurance which tells if a certain process has been carried out successfully. Taking advantage of IoT and its benefits to monitor cold chain logistics will result in better management and product handling. This paper looks at a system comprising of Arduino wireless sensor network and Xively sensor cloud which can be an ideal system to monitor temperature and humidity of cold chain logistics.
\end{abstract}

Keywords: Internet of Things; Wireless Sensor Network; Sensor Cloud; Cold Chain logistics; Arduino; Xbęe

\section{Introduction}

Cold Chain ogisties refers to the transportation of pharmaceutical products, biologics and active ingredients in controled temperature environment. These temperature sensitive items require such environment to the very last stage of the cold chain so that they do not lose their potency and is fit for use or consumption. This applies to both high-risk and low-risk products such as insulins, vaecines and blood products [1]. The counterfeit products are a real threat to public health and safety [2].

In the early 1990's, Food and Drug Administration (FDA) and U.S. Department of Agriculture (USDA) began to convey the Hazard Analysis Critical Control Point (HACCP) rules as a systematic approach to food safety [3]. The same principle has now been applied to other Industries dealing with temperature sensitive products such as the pharmaceutical industry. The HACCP management system addresses various circumstances for product safety and among them is the handling and distribution of the finished product. HACCP has become universally recognized and accepted standard for product safety and has been adopted by the WHO Standards Programme [4]. At every point in the cold chain, precautions are taken to ensure that the external conditions do not have any effect on the quality and stability of the products. Records of essential parameters such as temperature and humidity are maintained to provide evidence of compliance with recommended storage conditions.

The IoT technology ties the physical world objects to the Internet and gives the flexibility to easily monitor and manage them [5]. This body brings new perception to the way in which we interact with our environment on multiple levels including cultural, business, social, environmental, etc. [6]. The sensor entity which is emerging into IoT is wireless sensor 
network. Wireless sensor networks are spatially distributed autonomous sensors that monitor physical or environmental conditions. Applications of WSNs are in the areas such as structural health monitoring, health monitoring, environmental observation, habitat monitoring, and tracking among others [7]. These networks can be composed of thousands of small smart devices with computational capability and memory, one or more sensors and a limited power supply. The continuous progress in emerging sensor technologies such as WSNs has inspired IoT as this technology allows wide and cheap deployment of sensors on a large scale.

The integration of WSNs to the Internet needs high performance computing and storage infrastructure to perform real-time data processing and storage of data from WSNs and analyzing processed information to extract events of interest [8]. For this reason cloud computing is proving to be a promising technology to provide flexible computing, storage and software services in a scalable and virtualized manner [9]. Sensor cloud infrastructure [10] is a secondary form of cloud computing that has been proposed by severah IT people in present times. Sensor cloud allows managing of physical sensors on TT infrastructure. By utilizing the sensor cloud platform, several matters such as storing of collected data and processing them have become simple. Many real life applications are being assimilated to the sensor cloud including environmental monitoring, structural monitoring, disaster monitoring, agriculture, health care, telemetry, etc.

\section{Literature Review}

\subsection{Related Work}

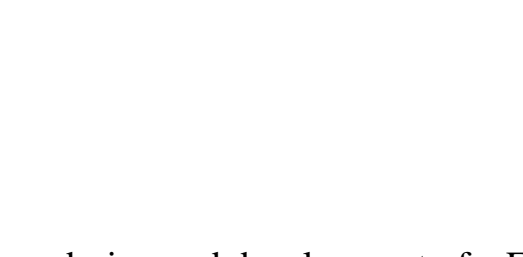

In [11], the authors describe a design and development of a Flexible Tag Datalogger (FTD) to improve food and goods logistics during transportation, storage and vending. The device is composed of three sensors (temperature, humidity and light) and a microcontroller. The sensor data is transmitted on an infrared communication (as an alternative to the RFID technology) to enable the communidation with most common personal devices such as smartphones or PDA with integrated infrared port. The device was tested on bottled wine by developing custom packaging to house the device to allow it to be easily wrapped around the bottleneck. The flexible tag collects data of its environment once the bottles leave the producer cellar for shop. When bottles arrive at the shop, the FTD attached to them downloads the data and is analyzed by PDA.

Performance of Z ZgBee-Based wireless sensor nodes has been studied in [12] for real-time monitoring of ruit logistics. WSNs are a promising solution to the monitoring applications and this work explores the potential of this technology for monitoring fruit in storage and transportation conditions. It focuses on two different commercial modules of ZigBee, Xbow and xbee. The experiments were carried out in experimental refrigerated chambers and the remainders in a commercial store. The main parameters considered were the ratio of measurement losses (\%), battery life (minimum) and the influence of node location and on/off operation of the cooling system.

Radio frequency identification (RFID) is an innovative and renowned technology that supports all types of traceability systems in many different areas and the work in [13] presents a temperature monitoring solution at the box level in the fish supply chain as part of the traceability system implemented using RFID technology. The temperature is measured in two ways; RFID data loggers are placed inside the box to measure temperature of the product and on the box to measure the ambient temperature. Sensor data is available immediately at the delivery by mobile RFID reader and afterwards stored in the database to be presented on web to stakeholders and private consumers. 
Work in [14] also looked at the performance of ZigBee motes through experiments under real conditions. The objective of the experiment was to test wireless sensor motes based on the ZigBee/IEEE 802.15.4 protocol during shipment of refrigerated vegetables. The experiment was conducted in a refrigerated truck travelling through Spain and France. The paper illustrated great potential of this type of motes that could provide information about several parameters such as temperature, relative humidity, opening of doors and truck stops.

\subsection{Technologies in this Work}

2.2.1. Internet of Things: In the beginning, the Internet was designed only for contputers so that they could access websites, download contents or communicate with other computers. The evolution of technology resulted in surfacing of powerful and faster devices with higher capabilities. With this evolution was the advancement of electronics technology that resulted in development of smaller devices which had high processing capabilities and low power consumption. Due to these devices, large networks of sensors can be created, having the ability to obtain and process information and act accordingly. This idea of connecting objects or things to the Internet goes back a few decades. The early inyentions of such devices were a toaster by John Romkey and Simon Hackett and a coffee pot by Quentin Stafford-Fraser and Paul Jardetzky in 1989 and 1992 respectively [15,16]. The term 'Internet of Things' was later coined by Kevin Ashton in 1999, the dirêctor of MIT Auto-W center, with the concept that computers could get information about the real world without any help from people and giving information [17].

2.2.2. Arduino: Arduino is an open-source electronics prototyping platform that is based on easy-to-use hardware and software. It is a board based platform with a single microcontroller input/output pin for communication and control of physical objects and a development environment for writing algorithms for the board. The platform grew largely out of "Do It Yourself" situation created by the proliferating popularity of rapid prototyping boards like PIC and Wiring, as well as the response to the growing needs of artists and designers to easily prototype interactive works (18). The Arduino devices have been developed to connect all sorts of objects and its functionality directly relates it to IoT.

2.2.3. Web 2.0: Web 20 is the second generation World Wide Web that is focused on people to have the ability to collaborate and share information online. Formerly, users could only view or download data contents posted on websites but with this new web, the users have more input into the nature and scope of the web contents and in some situations exert realtime control over it.

2.2.4. Mobile Data Network: $3 G$ mobile data network is the result of a decade of development and evolution of GSM standard to support increased data needs. It allows network operators to provide its users a bigger range of services due to bigger network capacity via heightened spectral efficiency. The network covers a wide area of cellular telephone networks which incorporates high-speed Internet connection and video telephony.

2.2.5. Near field Communication: Near Field Communication (NFC) is a wireless communication protocol which enables short-range communication between devices through high frequency to exchange data. Its root traces back to radio frequency identification (RFID). It is based on radio waves where data is exchanged between an identification card (NFC card) and a reader when brought together to a distance of about 4 centimeters. 
2.2.6. ZigBee: ZigBee is a communication protocol that uses small, low-power digital radios. It is based on the IEEE 802.15.4 standard for wireless personal area networks (WPANs) and is managed by the ZigBee Alliance. ZigBee is intended to be simpler and cheaper than other WPANs such as Wi-Fi and Bluetooth. This technology is targeted at radio-frequency (RF) applications which require low data rate, secure networking and longer battery life hence it is the most widely used standard in WSNs. Sensor data are typically a few tens of bytes therefore a high bandwidth is not necessary and ZigBee's low bandwidth helps in fulfilling the goal of low power, low cost and robustness [19].

\section{System Architecture}

The architecture of the proposed system for monitoring cold chain logistics Is shown in Figure 1. The system can be used in transport facilities that carry products from the manufacturing site to distribution center or from one distribution center to another or from a distribution center to the customer/consumer. The workflow of the monitoring system is as follows. Each package must have unique NFC tag to identify the products being transported.

The hub/Base Station of the system reads the products as they enter the truck. Inside the truck, Nodes are installed at multiple places containing temperature and humidity sensors. Multiple nodes allow for a more robust monitoning as it will cater for different temperature points in the vicinity. These nodes also include wireless communication function that allows it to consistently send temperature and humidity values to the base station. The base station gathers data from the nodes and prepanes them for upload to Sensor cloud together with GPS location of itself. The inventory data which is prepared from the NFC tags are emailed to the respective personnel. The location data is used to locate and trace the transport facility.

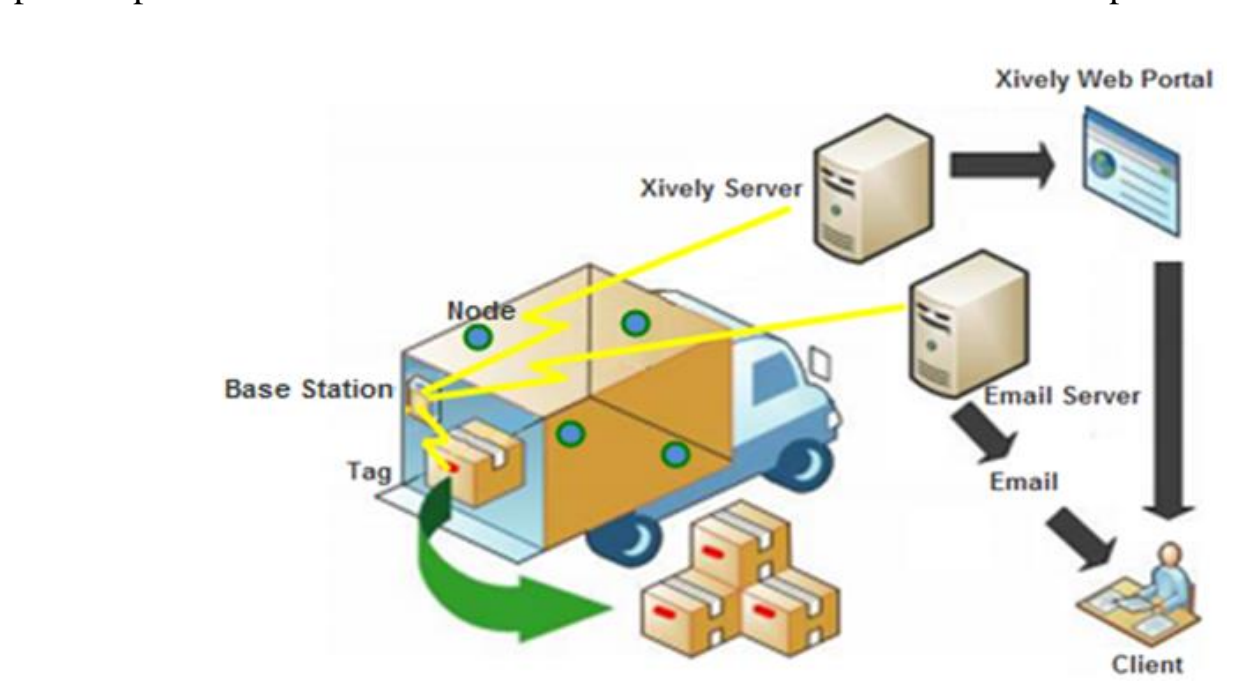

Figure 1. The Architecture of the System

\section{Implementation of the System}

In this section, we describe the implementation details of the proposed cold chain monitoring system for logistics [20]. We will focus on both the software and hardware development of the system as well as the configurations on the sensor cloud.

The hardware platform used for the system is Arduino. The overall components of the base station include Arduino microcontroller (Mega 2560), 3G+GPS shield for Arduino from Cooking Hacks, NFC shield from Adafruit, series2 Xbee shield and a battery. The sensor 
node consists of series 2 Xbee radio, one temperature and humidity sensor and a battery. Table 1 shows the specifications of the base station and sensor node. Figure 2 shows base station and sensor node deployed in the transport facility.

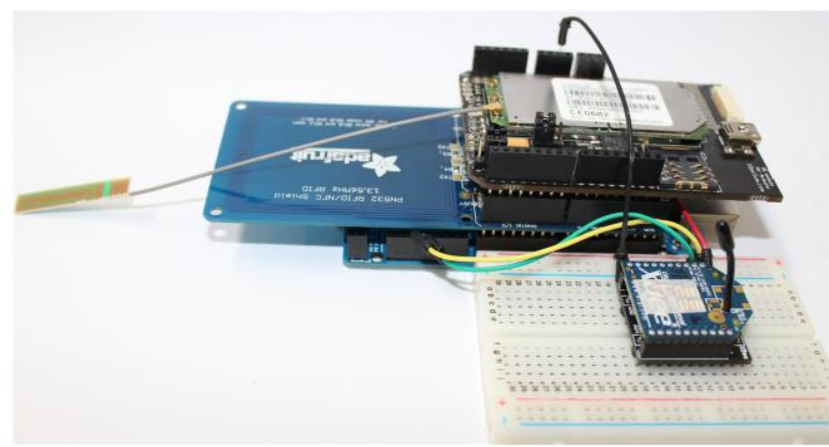

(a)

Figure 2. (a) Base Station (b) Node
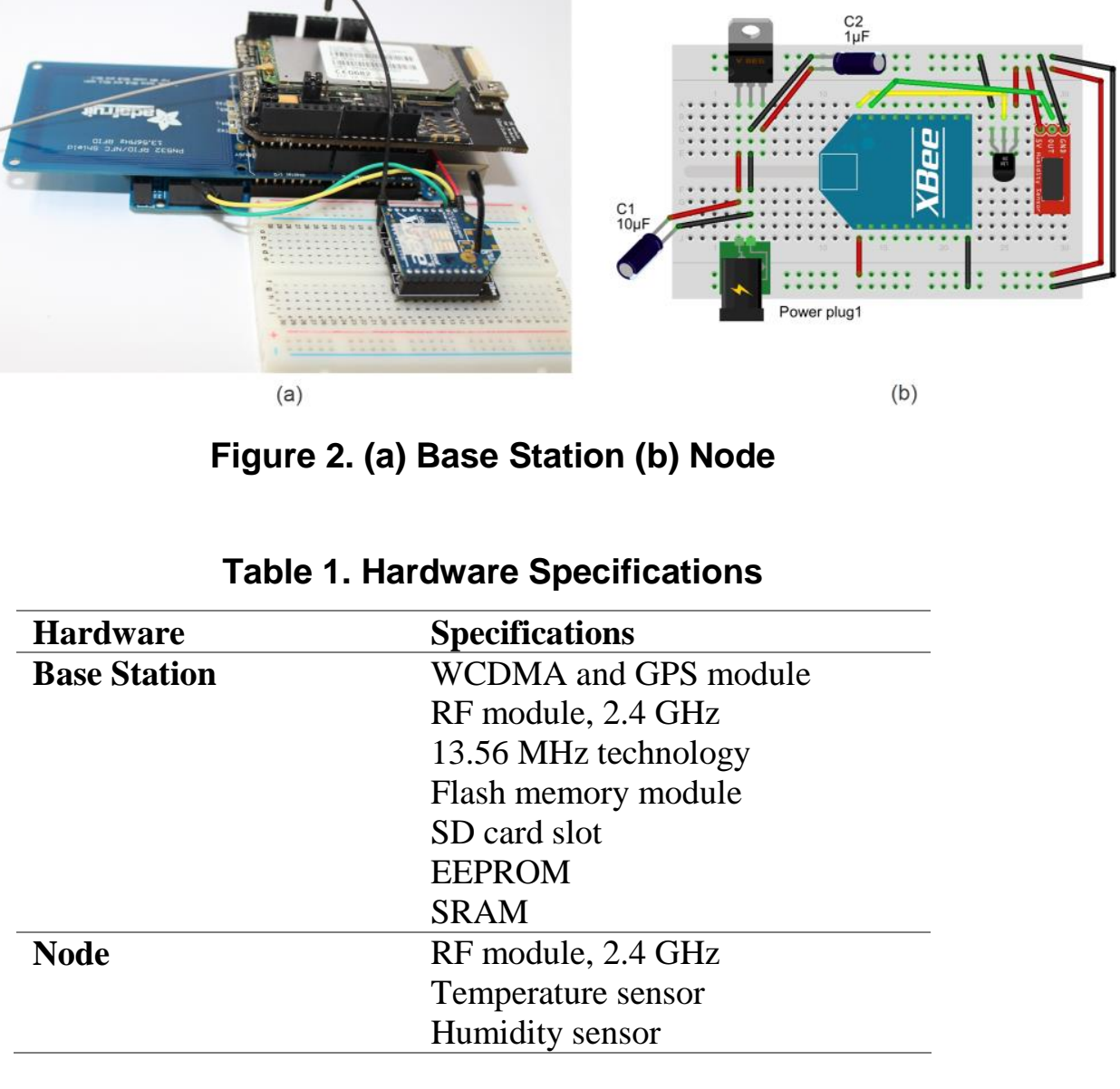

The Xbee radios have the capability to gather and transmit sensor data without the aid of an external microcontroller. Using Xbee for direct input or output reduces the overall size of the network, teduces power consumption, lowers the overall cost and presents less complicated approach. Series2 hardware allows its first four pins to be configured as analog input. First wo pins of the Xbee were configured to read the analog values of the temperature and humicity sensors. The nodes send their measured sensor values to the base station at fixed intervals.

The base station, after receiving data from a node, prepares the data so that it can be uploaded to the Xively cloud service. Xively sensor cloud is an open platform that provides service to its users free of charge. When creating a new device on the service (Refrigeration Chamber), Feed ID and API key is auto-generated to be used in Arduino program for uploading data to that feed. The feed contains a collection of datastreams for each node in the monitoring environment of the transport. Developer bench of Xively is used for visualizing the incoming sensor data as well as the metadata that is the device location. Figure 3 shows the developer workbench of Xively. The additional datastream is used for monitoring the supply voltage of the nodes to be aware of the battery status. 


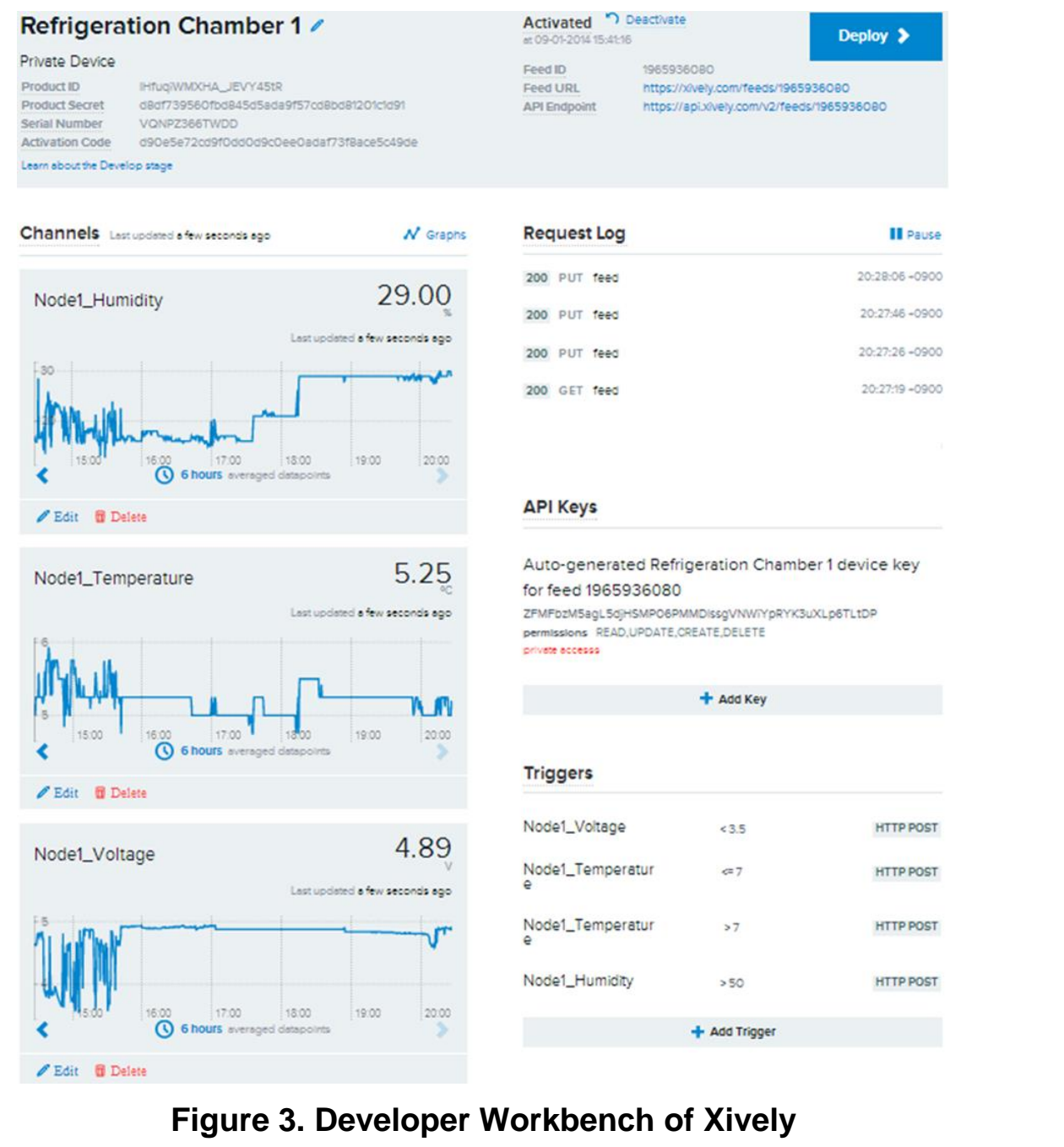

On Xively, the senordata are graphed for easy visualization. The triggers are one of the important components as it allows setup of notification when certain events occur. These notifications are linked to email address(s) of appropriate personnel to take evasive actions during times these events occur. Zapier is an important application for this task as it allows automating tasks between web applications and they are Xively and email service. We define the events as shown in Table 2. For this experimentation, temperature range of 2-8 degrees Celsius 15 considered as the safe zone for products and a humidity value below 50 percent. Email notification is sent when any of the condition is met. Figure 4 depicts the email notification sent by Xively as well as email generated at base station regarding the inventory in the transport facility.

Table 2. Criterions for Trigger

\begin{tabular}{ll}
\hline eadings & Event \\
\hline Temperature $>\mathbf{7}$ & Temperature above maximum threshold \\
\hline Temperature $<=\mathbf{7}$ & Temperature returned to normal \\
\hline Temperature $>\mathbf{= 3}$ & Temperature returned to normal \\
\hline
\end{tabular}




\begin{tabular}{ll}
\hline Temperature $<\mathbf{3}$ & Temperature below minimum threshold \\
\hline Humidity $>\mathbf{5 0}$ & Humidity above maximum threshold \\
\hline Humidity $<\mathbf{5 0}$ & Humidity returned to normal \\
\hline Voltage $<\mathbf{3 . 5}$ & Node voltage low \\
\hline
\end{tabular}

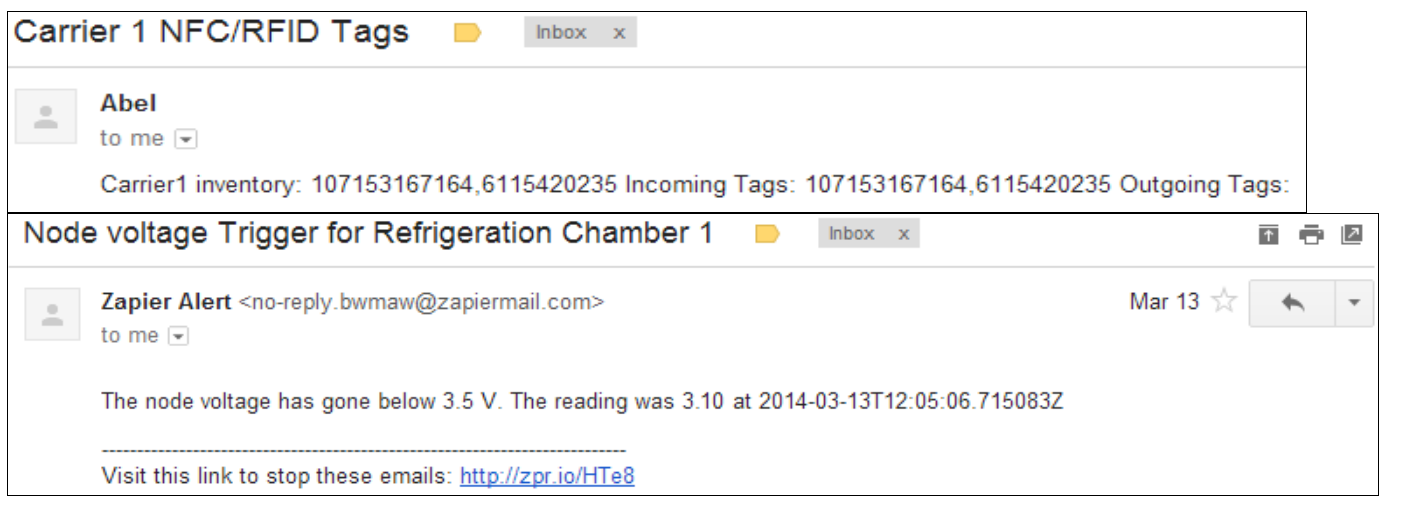

Figure 4. Email Notification from Xively and Inventory Information by Email

\section{Conclusion}

The expansion of embedded IC Infrastrueture has caused the deployment of wide range of embedded systems in our environment which points out the need for manageable and flexible wireless sensor network. IoT is a solution which proves to be a promising concept. We proposed the for concept for monitoring cold chain logistics that offers an easy to /manage and reautime monitoring using WSN and sensor cloud. With the integration of WSN infrastructure to cloud, there are numerous benefits where common processing computational and analytical tasks can be hosted on the cloud service rather than the WSN itself therefore freeing the device from running heavy algorithms and programs hence reducing power consumption and increasing the network lifetime. The data visibility also increases which enhances the monitoring and management of wireress sensor networks.

\section{Acknowledgements}

This work was supported by Basic Science Research Program through the National Research Eoundation of Korea(NRF) funded by the Ministry of Education (NRF-20090093828) and the MSIP (Ministry of Science, ICT and Future Planning), Korea, under the CITRC (Convergence Information Technology Research Center) support program (NIPA2014-H0401-14-1009) supervised by the NIPA (National IT Industry Promotion Agency).

\section{References}

[1] J. Tennermann, Cold Chain for Beginners. Available: http://www.pharmpro.com/articles/2012/06/cold-chainbeginners, (2012).

[2] "WHO good distribution practices for pharmaceutical products," World Health Organization2010.

[3] W. Stilmant, "Pharmaceutical Cold Chain", Available: http://www.coldchain.be/guidelines-andregulations/some-history.html, (2013).

[4] Hazard Analysis Critical Control Point System $\quad$ (HACCP). Available: http://www.who.int/foodsafety/fs_management/haccp/en/, (2007). 
[5] M. C. Domingo, "An overview of the Internet of Things for people with disabilities", Journal of Network and Computer Applications, vol. 35, no. 2, (2012), pp. 584-596.

[6] L. Atzori, A. Iera and G. Morabito, "The Internet of Things: "The Internet of Things: A survey," Computer Networks, vol. 54, no. 15, (2010) October, pp. 2787-2805.

[7] I. Khemapech, I. Duncan, and A. Miller, "A Survey of Wireless Sensor Networks Technology", in $6^{\text {th }}$ Annual Postgraduate Symposium on the Convergence of Telecommunications, Networking and Broadcasting, Liverpool, UK, (2005) June.

[8] J. K. Hart and K. Martinez, "Environmental Sensor Networks: A revolution in the earth system science? Earth-Science Reviews", vol. 78, no. 3, (2006), pp. 177-191.

[9] J. Peng, X. Zhang, Z. Lei, B. Zhang, W. Zhang and Q. Li, "In Comparison of several cloud computing platforms", Information Science and Engineering (ISISE), 2009 Second International Symposium on, IEEE: (2009), pp 23-27.

[10] M. Yuriyama and T. Kushida, "In Sensor-cloud infrastructure-physical sensor management with virtualized sensors on cloud computing", Network-Based Information Systems (NBiS), 2010 13th International Conference on, IEEE: (2010), pp 1-8.

[11] V. Mattoli, B. Mazzolai, A. Mondini, S. Zampolli, and P. Dario, "Flexible tag datalogger for food logistics", Sensors and Actuators A: Physical, vol. 162, (2010), pp. 316-323,

[12] L. Ruiz-Garcia, P. Barreiro, and J. Robla, "Performance of ZigBee-based wireless sensot nodes for real-time monitoring of fruit logistics", Journal of Food Engineering, vol, 87, (2008), pp. 405-415.

[13] M. Trebar, M. Lotrič, I. Fonda, A. Pleteršek, and K Kovacic, "RFWD Data Loggers in Fish Supply ChainTraceability", International Journal of Antennas and Propagation, ol. (2013).

[14] L. Ruiz-Garcia, P. Barreiro, J. I. Robla, and L. Lunadei, "Testing ZigBee motes for monitoring refrigerated vegetable transportation under real conditions", Sensors, yol. 10, (2010), pp. 4968-4982.

[15] "Australian UNIX systems User Group Newsletter", AUUG Incorporated, vol. 12, p. 54, (1991).

[16] Q. S. Fraser, "The Trojan Room Coffee Pat", Available: http://www.cl.cam.ac.uk/coffee/qsf/coffee.html, (1995).

[17] K. Ashton, "That 'Internet of Things' Thing", Available: bttp://www.rfidjournal.com/articles/view?4986, (2009).

[18] K. Leung, “Arduino: A Brief Historyl, Available: http://www.kenleung.ca/portfolio/arduino-a-brief-history$3 /$

[19] G. Legg, “ZigBee: Wireless Technology for Low-Power Sensor Networks”, Available: http://www.eetimes.com/document.asp?doc/id=1275760, (2004).

[20] A. Chandra, K. Kim, S. Kin,, and S. Lee, "Advanced Monitoring of Cold Chain Logistics using WSN and Sensor Cloud Infrastructure", in Liternational Conference on Future Information \& Communication Engineering, Hong Kong, (2014).
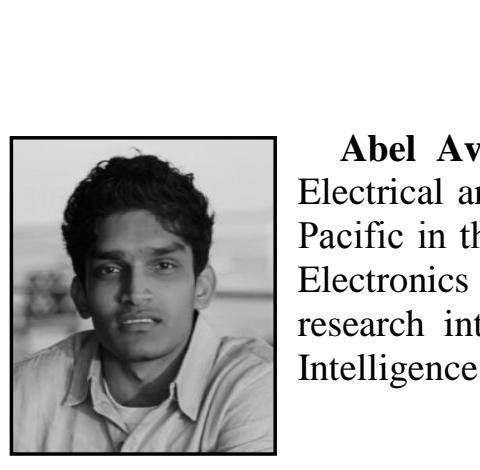

\section{Authors}

Abel Avitesh Chandra, he has obtained his Bachelor's degree in Electrical and Electronics Engineering from the University of the South Pacific in the year 2011. He is currently a Master's student majoring in Electronics and Communication at Mokpo National University. His research interest is in the areas of Machine Learning, Computational Intelligence, and Wireless Sensor Networks.

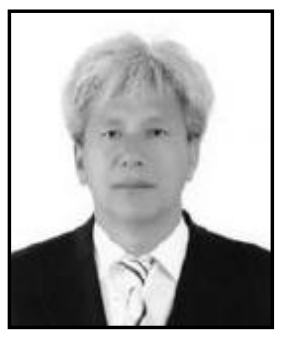

이성로 (Seong Ro Lee)

1987년 2월:고려대학교 전자공학과 공학사

1990년 2월: 한국과학기술원 전기 및 전자공학과 공학석사

1996년 8월: 한국과학기술원 전기 및 전자공학과 공학박사

1997년 9월 현재:목포대학교 공과대학 정보전자공학과 교수

<관심분야> 디지털통신시스템, 이동 및 위성통신시스템, USN/텔레미틱스응용분야, 임베디드시스템 